\title{
Radiotherapy of the Lymphatic Pathways in Early Breast Cancer
}

\author{
Marie-Luise Sautter-Bihla Felix Sedlmayer ${ }^{b}$ \\ ${ }^{a}$ Klinik für Radioonkologie und Strahlentherapie, Städtisches Klinikum Karlsruhe, Germany; \\ ${ }^{b}$ Department of Radiotherapy and Radiation Oncology, LKH Salzburg, Paracelsus Medical University Hospital, Salzburg, Austria
}

\section{Keywords}

Breast cancer · Radiotherapy · Regional nodal irradiation

\section{Summary}

International guidelines reveal substantial differences regarding indications for regional nodal irradiation (RNI). Recently, several randomized studies provided new insights and these are discussed here. Patients with 1-3 positive nodes seem to profit from RNI compared to whole-breast (WBI) or chest-wall irradiation (CWI) alone, both with regard to locoregional control and disease-free survival. Irradiation of the regional lymphatics including axillary, supraclavicular and internal mammary nodes provided a small but significant survival benefit in recent randomized trials and 1 meta-analysis. Lymph node irradiation yields comparable tumor control in comparison to axillary lymph node dissection while reducing the rate of lymph edema. Data concerning the impact of 1-2 macroscopically affected sentinel nodes or microscopic metastases on prognosis are equivocal. Recent data suggest that the current restrictive use of RNI should be scrutinized, as the hazard-benefit relation appears to shift towards an improvement of outcome.

(c) 2015 S. Karger GmbH, Freiburg

\section{Introduction}

Radiotherapy (RT) of the regional lymph node (LN) areas in early breast cancer has become an issue of controversy over the past few decades as the awareness of late side effects has increased. Although irradiation of the complete lymphatic pathways was part of the treatment in almost all studies investigating post-mastec- tomy RT (PMRT) [1], it was only used in a minority of patients after breast-conserving surgery (BCS) [2]. For regional nodal irradiation (RNI), older studies generally included RT of the supraclavicular (SCNs), axillary (ALNs) as well as internal mammary (IMNs) nodes. More recently, the use of IMN RT has substantially declined as it was suspected to enhance cardiac toxicity and morbidity [3]. Similarly, dedicated RT to the axilla is now considered obsolete for pathologically node-negative patients, assessed by either ALN dissection (ALND) or sentinel node (SN) biopsy (SNB), and even for selected SN-positive patients.

The aim of the present paper is to provide an overview on current literature and to highlight different aspects concerning indications for RNI. The recent recommendations of the German Society for Radiation Oncology (DEGRO) [4] based on lately published randomized trials are discussed.

\section{RNI: Definition of a Plurivalent Term}

The term RNI comprises a spectrum of different target volumes. The SCNs are mostly included in RNI; however, the target volume is not strictly defined. Some investigators include the medial SN only [5], while others extend the lateral field border to the neck of the humerus [6]. The term 'infraclavicular nodes' (also widely used to describe 1 part of SCN RT) is identical to level III of the ALNs. Even though these nodes are predominantly not an explicit part of the treatment plan, they are mostly included in order to avoid a gap between SCNs and the tangential whole-breast irradiation (WBI) fields. Even when the ALNs are explicitly not part of the target volume for WBI, a significant portion of leveI I is inadvertently included [7]. The same holds true for the IMNs where substantial doses of the tangential fields may be deposited. Before the era of CT-based 3 dimensional (3-D) contouring of the defined LN regions, unintended irradiation of these areas may have contributed to the reduction of locoregional recurrences, however to a non-

\section{KARGER \\ Fax +497614520714

\section{(c) 2015 S. Karger GmbH, Freiburg}

$1661-3791 / 15 / 0104-0254 \$ 39.50 / 0$
Prof. Dr. med. Marie-Luise Sautter-Bihl 
quantifiable extent. Thus, recommendations for irradiation of the different LN areas are not evidence based in a strict sense, as RT of the SCNs as an isolated mode of nodal irradiation has not been investigated in randomized studies (an exception is described below).

\section{General Indications for RNI}

Until recently, no randomized studies explicitly investigated the quantitative benefit of RNI in addition to postoperative RT of the breast or chest wall. Nonetheless, if 4 positive ALNs are present, the indication for RNI has been undisputed, although some controversy remained for patients with 1-3 positive nodes ( $\mathrm{pN1}$ ) or tumors of $>5 \mathrm{~cm}[4,8-10]$. Indirect evidence for the benefit of RNI for patients with 1-3 positive ALNs was derived from subgroup analyses of the Danish Breast Cancer Group study and the British Columbia trial. Both randomized studies yielded an improvement of survival when PMRT, including the complete lymphatic pathways, was used. Comparison of outcomes according to the number of metastatic nodes revealed that patients with 1-3 and those with $\geq 4$ positive nodes had a similar absolute overall survival (OS) advantage of roughly $10 \%[11,12]$. As no comparison was made to chest-wall irradiation (CWI) alone, uncertainty remained as to how to quantify the contribution of RNI.

\section{Benefit of RNI for Patients with 1-3 Positive Nodes}

The first randomized study providing evidence for the benefit of RNI especially in patients with 1-3 LN after BCS was the NCICCTG MA.20 trial [6]. The study comprised 1,832 women with mostly $1-3$ positive ALNs (85\%) and a minority of women (10\%) with negative nodes but possessing high-risk factors. Patients were randomized after BCS and ALND to either WBI or WBI and additional RNI. The 10-year locoregional recurrence-free survival was 95.2\% with and $92.2 \%$ without RNI $(\mathrm{p}=0.009)$. 10-year diseasefree survival (DFS) was significantly improved in the RNI group: $82 \%$ vs. only $77 \%$ patients with WBI alone $(\mathrm{p}=0.01)$. This difference is higher than the absolute benefit in terms of local control, and therefore hypothetically attributable to the significant positive impact on distant metastases-free survival with an absolute 3.9\% reduction at 10 years in the $\mathrm{RNI}$ arm $(\mathrm{p}=0.03)$. There was no significant difference in OS (82.8 vs $81.8 \%$ ). The rate of lymph edema (any grade) was $4.5 \%$ without compared to $8.4 \%$ with RNI ( $\mathrm{p}=0.001$ ), which is in accordance to recent literature [13]. The authors concluded that RNI reduces the risk of locoregional and distant recurrence but does not improve OS [6].

\section{Benefit of RNI for pNO and pN1(-2)}

The EORTC 22922-10925 trial [5] included 4,004 women diagnosed as stage I-III with mostly pT1-2 tumors (95\%), and involved ALNs (55.6\%) and/or a medially located primary tumor (44.5\%).
The majority of node-positive women had 1-3 involved ALNs. Patients were randomized after BCS (76.1\%) or mastectomy (23\%) to receive $\mathrm{RT}$ to the breast or chest wall with or without inclusion of the IMN and medial SCN (MSCN). Dose specifications for WBI/ CWI were not required, and presumably, relevant parts of the axilla were included in these fields. Nearly all LN-positive (99.0\%) and $66.3 \%$ of the LN-negative patients received adjuvant systemic treatment. After adjustment for stratification factors, RT to the IMN and MSCN chain significantly improved outcome at 10 years: OS: 82.3 vs. $80.7 \%, \mathrm{p}=0.049$; DFS: 72.1 vs. $69.1 \%, \mathrm{p}=0.044$; metastases-free survival: 78.0 vs. $75.0 \%, p=0.020$. The treatment effect on OS was similar for $\mathrm{pN} 1$ and $\mathrm{pN} 2$ patients but, interestingly, most pronounced for node-negative patients (hazard ratio (HR) $0.79,95 \%$ confidence interval (CI) $0.61-1.02$ ). The highest benefit was observed in patients receiving chemo- as well as endocrine therapy (HR 0.72, 95\% CI 0.55-0.94). While the local recurrence rate was similar (5.3 vs.5.6\%), regional $\mathrm{LN}$ recurrence was $2.7 \%$ with RNI vs. $4.2 \%$ without. In contrast to the findings in the Canadian study [6], the rates of any-grade lymph edema at 3 years were identical in both groups [14]. No increase in lethal complications has been observed so far. Therefore, the authors concluded that RT of the IMN and MSCN should be recommended for patients with involved ALNs and/or medially located primary tumor.

A meta-analysis of these data [15] revealed an even more distinct benefit of RNI on OS with an HR of $0.82(\mathrm{p}=0.011)$. The largest gain was observed for distant metastases-free survival, possibly supporting the hypothesis of Hellmann [16] that RT is 'stopping metastases at their source'.

\section{Benefit of IMN RT?}

The restricted use of IMN RT is triggered by the fear of enhanced cardiac toxicity $[3,17]$. Indeed, an increase of cardiovascular-related mortality was observed in older studies [1]. Undisputedly, IMN RT increases the dose to the heart, even with sophisticated 3-D planning or intensity-modulated RT (IMRT) however, much less than formerly achievable $[18,19]$. The rationale to include the IMNs was originally based on autopsy series in the 1960s showing a high percentage of metastatic IMN [18], which has more recently been confirmed in a large series of 1,679 Chinese women who underwent extended mastectomy including dissection of the IMNs. Patients with 4 or more positive ALNs and medial tumor or any positive ALNs, T3 tumors and an age less than 35 years had an increased (more than 20\%) risk for IMN metastases [20]. Veronesi et al. [21] performed several studies addressing local treatment of the IMN. In a randomized study of 737 patients, dissection of the IMNs did not improve survival in comparison to mastectomy alone. In a further clinical study of 68 patients receiving RT of the IMN for histologically proven metastases, RT was highly effective and yielded a 5-year OS of 95\% [22].

Besides restriction of IMN RT because of the fear of cardiovascular-related mortality [17], another argument against IMN RT was the small number of patients diagnosed with clinically mani- 
fest IMN recurrence which is assumed to be only about $1 \%$ [18]. A possible explanation is the lack of imaging of this area as part of the follow-up program. Moreover, parts of the IMN may be inadvertently included in tangential field arrangements. Another hypothesis is that micro-metastases in the IMNs may represent a source for metastatic spread without growing to a clinically detectable size before distant metastases were diagnosed [15].

Only one study selectively addressed the effect of IMN RT in comparison to PMRT and SCN RT. The French trial [23] included 1,334 patients with mostly T1-2 tumors ( $85 \%$ ) and either positive ALNs (75\%) or central/medial tumors irrespective of nodal status (25\%). All patients were treated with PMRT to the chest wall, including SCN (plus axillary apex, in node-positive cases) and were randomized to receive additional IMN RT or not. Roughly $60 \%$ received chemotherapy, about $50 \%$ endocrine systemic treatment. Overall, 10-year OS was $62.57 \%$ with IMN RT and 59.3\% (n.s.) without. Node-negative patients $(25 \%)$ showed a trend towards a worse outcome with IMN RT (n.s.), whereas node-positive patients seemed to profit from IMN RT (n.s.). The authors acknowledged that the study may have been underpowered for proving a significant survival benefit for IMN RT. No increase in cardiac toxicity was observed in the IMN RT group. The authors stated that their data did not permit a definite conclusion; therefore, they could not reliably recommend for or against IMN RT [23].

\section{Positive SN/No ALND - Implications for RT}

A new challenge for the radiation oncologist emerged with a change of practice in axillary surgery [24]. Based on data of a randomized study of the American College of Surgeons Oncology Group [25], several guidelines [8, 9] even permit omission of ALND in selected patients with 1 or 2 pathologically positive nodes after BCS, provided they receive adjuvant WBI. The ACOSOGZ0011 study was a randomized noninferiority trial, including women with stage I or stage IIA breast cancer with clinically negative axilla who underwent SNB that revealed 1-2 pathologically affected nodes. Overall, 891 patients were randomized to either ALND $(n=445)$ or no further local treatment $(n=446)$. All patients received adjuvant WBI. After a median follow-up of 6.3 years, no difference in OS and DFS was observed; 5-year locoregional recurrence-free survival rate was $96.7 \%$ after SNB alone and $95.7 \%$ in patients with ALND. The authors concluded that ALND may no longer be justified in patients with T1-2 tumors.

Unexpected insights about the quality of evidence generated by the Z0011 trial were provided by a recent publication on behalf of the Alliance for Clinical Trials in Oncology. Jagsi et al. [26] attempted to analyze the radiation field design of the Z0011 study population by requesting the treatment records from the involved radiation oncologists for a central review. Completed case report forms were obtained for 605/856 patients, and of those 540 (89\%) had received WBI. Additional treatment to the supraclavicular region was recorded in $89(15 \%)$ of these patients in this subgroup. Of note, detailed RT records were only available for 228 patients, of whom 185 (81.1\%) received tangents alone. These findings demonstrate the total lack of standardization or quality control in the Z0011 study, therefore precluding ultimate conclusions with regard to local treatment of the axilla [27]. Further insights concerning the adequate approach for the clinically negative axilla, even including abandonment of SNB, is awaited from the ongoing INSEMA trial [28].

A compromise accounting for a potentially increased risk of locoregional recurrence of $\mathrm{SN}$-positive patients was proposed by Haffty et al. [24], who suggested the use of 'high tangents', with the rationale of including the nodal area presumed to be at highest risk. This approach was based on several studies investigating the dose delivered to the ALNs by conventional tangential fields, and the exploration of techniques yielding an improved coverage of level I by minor field extensions in cranial direction [29-31]. This technique is also recommended in the new textbook on Radiation Oncology edited by Perez and Brady [18]. However, this approach is neither based on solid data, nor does it address a clearly defined, 3-D-rendered target volume as demanded in modern RT.

\section{Is ALN RT the Alternative to ALND for SN-Positive Patients?}

Recently, the EORTC 10981-22023 AMAROS study [32] investigated the effectiveness of ALN RT (including SCN) in comparison to ALND. Overall, 4,806 patients with clinically negative nodes received SNB. Patients with a negative SNB did not receive any axillary treatment (except WBI) and the 5-year rate of axillary recurrence was $0.8 \%$. SNB was pathologically positive in 1,425 patients, who were randomized for either ALN RT or ALND. Of the latter, $60 \%$ showed macro-metastases (>2 $\mathrm{mm}$ ). In the ALND group, $67 \%$ had no further positive nodes; $1-3$ affected nodes were found in $25 \%$ and $>4$ affected nodes in $7.8 \%$. Subgroup analyses were not presented. The 5-year axillary recurrence rate was not significantly different: $0.43 \%$ after ALND and 1.19\% after ALN RT. The planned noninferiority test was underpowered because of the unexpectedly low number of events. No significant differences in OS (93.2\% vs. $92.5 \%, \mathrm{p}=0.33)$ and DFS ( $86.9 \%$ vs. $82.6 \%, \mathrm{p}=0.17)$ were observed. Lymph edema (any grade, including minor circumferential differences) was found significantly more often after ALND: 5-year rate $28 \%$ compared to ALN RT $14 \%(\mathrm{p}=0.0001)$. Considering that the pattern of spread was equally distributed in both groups, it can be assumed that in the ALN RT group roughly a third had remnant axillary metastases after SNB. The low recurrence rate of $1.19 \%$ strongly indicates the comparable effectiveness of ALN RT as a less invasive procedure than ALND. Of note, patients who had undergone $\mathrm{BCS}$ received WBI, including parts of the axilla. The outcome of patients who received mastectomy and no irradiation may provide insights concerning the consequences of truly refraining from any local treatment of the axilla, but no such subgroup analysis has yet been presented. The authors concluded that ALN RT can be 'considered standard' in SN-positive patients [32]. 


\section{Targeting, Technique and Dose for RNI}

3-D treatment planning is mandatory, and several anatomically based instruction guidelines have been published to define individual contouring of the different $\mathrm{LN}$ regions [33-35].

Hypofractionation (HF) is currently not recommended for patients who receive RNI, as larger doses per fraction may increase the risk of long-term effects such as cardiac toxicity or plexopathy [36-38]. In the randomized studies investigating HF, 79\% of the patients were node negative, only a minority of patients were treated with RNI (Canadian study: none, START A trial: 13\%, START B trial: 7\% [39, 40]). The same applies for HF after chemotherapy [41], as the majority of HF patients had not received such treatment $[39,40]$ and most patients considered for RNI are nowadays planned to receive chemotherapy.

\section{Comments and Conclusions}

Data from the MA- 20 and EORTC studies suggest that all node-positive patients profit from comprehensive RNI including SCN and IMN. The respective contribution of RNI by site (SCN vs. IMN) on improved outcome cannot be distinguished; ALNs of level I and II were partly included in the control arms using WBI/ CWI. No increase in cardiovascular toxicity due to RNI has been reported. However, the follow-up 10 years in the three studies may still be too short for a final assessment of cardiotoxicity. Further follow-up and subgroup analyses have to be awaited.

The precept that IMN RT should not be performed has to be scrutinized and medial tumor location should regain relevance among decision criteria for IMN RT as part of RNI. RNI seems to yield a benefit for selected patients with $\mathrm{pN} 0$ stage and specific risk constellations or medial/central tumor location, but current data are conflicting and standardized recommendations for RNI in node-negative women cannot be derived.

Data do not yet permit ultimate conclusions as to whether any local treatment of the axilla can be safely omitted in selected patients with 1-2 involved LNs or in case of micrometastases. If macroscopic SN metastases exist, ALN RT (as part of RNI) should be discussed as an alternative to ALND in light of the equivalent effectiveness and concurrent reduction of lymph edema.

\section{Disclosure Statement}

The authors declare no conflicts of interest.

\section{References}

1 Clarke M, Collins R, Darby S, et al.: Effects of radiotherapy and of differences in the extent of surgery for early breast cancer on local recurrence and 15-year survival: An overview of the randomised trials. Lancet 2005;366:2087-2106.

2 Early Breast Cancer Trialists' Collaborative Group (EBCTCG), Darby S, McGale P, et al.: Effect of radiotherapy after breast-conserving surgery on 10-year recurrence and 15-year breast cancer death: Meta-analysis of individual patient data for 10,801 women in 17 randomised trials. Lancet 2011;378:1707-1716.

3 Darby SC, Ewertz M, McGale P, et al.: Risk of ischemic heart disease in women after radiotherapy for breast cancer. N Engl J Med 2013;368:987-998.

4 Sautter-Bihl ML, Sedlmayer F, Budach W, et al.: DEGRO practical guidelines: Radiotherapy of breast cancer III-radiotherapy of the lymphatic pathways. Strahlenther Onkol 2014;190:342-351

5 Poortmans PM, Collette S, Kirkove C, et al.: Internal mammary and medial supraclavicular irradiation in breast cancer. N Engl J Med 2015;373:317-327.

6 Whelan T, Olivotto I, Parulekar WR, et al.: Regional nodal irradiation in early breast cancer. N Engl J Med 2015;373:307-316.

7 Nitsche M, Temme N, Forster M, et al.: Tangential vs. defined radiotherapy in early breast cancer treatment without axillary lymph node dissection: a comparative study. Strahlenther Onkol 2014;190:715-721.

8 S3, Interdisziplinäre S3-Leitlinie für die Diagnostik, Therapie und Nachsorge des Mammakarzinoms, Langversion 3.0, Aktualisierung 2012, AWMF-Register-Nummer: 032-045OL 2012.

9 NCCN, Clinical Practice Guidelines in Oncology: Breast Cancer, Version 3.2013. National Comprehensive Cancer Network, 2013. www.nccn.org/professionals/physician_gls/f_guidelines.asp\#site.
10 Wenz F, Sperk E, Budach W, et al.: DEGRO practical guidelines for radiotherapy of breast cancer IV: Radiotherapy following mastectomy for invasive breast cancer. Strahlenther Onkol 2014;190:705-714.

11 Overgaard M, Nielsen HM, Overgaard J, Is the benefit of postmastectomy irradiation limited to patients with four or more positive nodes, as recommended in international consensus reports? A subgroup analysis of the DBCG 82 b\&c randomized trials. Radiother Oncol 2007;82:247-253.

12 Ragaz J, Olivotto IA, Spinelli JJ, et al.: Locoregional radiation therapy in patients with high-risk breast cancer receiving adjuvant chemotherapy: 20-year results of the British Columbia Randomized Trial. J Natl Cancer Inst 2005;97:116-126.

13 Shah C, Wilkinson JB, Baschnagel A, et al.: Factors associated with the development of breast cancer-related lymphedema after whole-breast irradiation. Int J Radiat Oncol Biol Phys 2012;83:1095-1100.

14 Matzinger O, Heimsoth I, Poortmans P, et al.: Toxicity at three years with and without irradiation of the internal mammary and medial supraclavicular lymph node chain in stage I to III breast cancer (EORTC trial 22922/10925). Acta Oncol 2010;49:24-34.

15 Budach W, Kammers K, Boelke E, et al.: Adjuvant radio-therapy of regional lymph nodes in breast cancer - a meta-analysis of randomized trials. Radiat Oncol 2013;8:267.

16 Hellman S, Stopping metastases at their source. N Eng J Med 1997;337:996-997.

17 Ceilley E, Jagsi R, Goldberg S, et al.: Radiotherapy for invasive breast cancer in North America and Europe: Results of a survey. Int J Radiat Oncol Biol Phys 2005; 61:365-373.

18 Goyal S, Buchholz T, Haffty B: Breast Cancer: Early Stage. In: Perez and Brady's Radiation Oncology, 6th edn. 2013.
19 Rudat V, Alaradi AA, Mohamed A, et al.: Tangential beam IMRT versus tangential beam 3D-CRT of the chest wall in postmastectomy breast cancer patients: A dosimetric comparison. Radiat Oncol 2011;6:26.

20 Huang O, Wang L, Shen K, et al.: Breast cancer subpopulation with high risk of internal mammary lymph nodes metastasis: Analysis of 2,269 Chinese breast cancer patients treated with extended radical mastectomy. Breast Cancer Res Treat 2008;107:379-387.

21 Veronesi U, Marubini E, Mariani L, et al.: The dissection of internal mammary nodes does not improve the survival of breast cancer patients. 30-year results of a randomised trial. Eur J Cancer 1999;35:1320-1325.

22 Veronesi U, Arnone P, Veronesi P, et al.: The value of radiotherapy on metastatic internal mammary nodes in breast cancer. Results on a large series. Ann Oncol 2008;19:1553-1560.

23 Hennequin C, Bossard N, Servagi-Vernat S, et al.: Tenyear survival results of a randomized trial of irradiation of internal mammary nodes after mastectomy. Int J Radiat Oncol Biol Phys 2013;86:860-866.

24 Haffty BG, Hunt KK, Harris JR, et al.: Positive sentinel nodes without axillary dissection: implications for the radiation oncologist. J Clin Oncol 2011;29:4479-4481.

25 Giuliano AE, Hunt KK, Ballman KV, et al.: Axillary dissection vs no axillary dissection in women with invasive breast cancer and sentinel node metastasis: A randomized clinical trial. JAMA 2011;305:569-575.

26 Jagsi R, Chadha M, Moni J, et al.: Radiation field design in the ACOSOG Z0011 (Alliance) Trial. J Clin Oncol 2014;32:3600-3606.

27 Sautter-Bihl ML, Sedlmayer F, Budach W, et al.: How nescience may obscure evidence. Strahlenther Onkol 2014;190:861-863. 
28 Reimer T, Hartmann S, Stachs A, et al.: Local treatment of the axilla in early breast cancer: Concepts from the national surgical adjuvant breast and bowel project B-04 to the planned intergroup sentinel mamma trial. Breast Care (Basel) 2014;9:87-95.

29 Alço G, Iğdem S, Ercan T, et al.: Coverage of axillary lymph nodes with high tangential fields in breast radiotherapy. Br J Radiol 2010;83:1072-1076.

30 Reznik J, Cicchetti MG, Degaspe B, et al.: Analysis of axillary coverage during tangential radiation therapy to the breast. Int J Radiat Oncol Biol Phys 2005;61:163168.

31 Schlembach PJ, Buchholz TA, Ross MI, et al.: Relationship of sentinel and axillary level I-II lymph nodes to tangential fields used in breast irradiation. Int J Radiat Oncol Biol Phy 2001;51:671-678.

32 Rutgers EJ, Donker M, Straver ME, et al.: Radiotherapy or surgery of the axilla after a positive sentinel node in breast cancer patients: Final analysis of the EORTC AMAROS trial (10981/22023). J Clin Oncol 2013 31:abstr LBA1001.
Dijkema IM, Hofman P, Raaijmakers CPJ, et al.: Locoregional conformal radiotherapy of the breast: Delineation of the regional lymph node clinical target volumes in treatment position. Radiother Oncol 2004;71: 287-295.

34 Offersen BV, Boersma LJ, Kirkove C, et al.: ESTRO consensus guideline on target volume delineation for elective radiation therapy of early stage breast cancer. Radiother Oncol 2015;114:3-10

35 Verhoeven K, Weltens C, Remouchamps V, et al.: Vessel based delineation guidelines for the elective lymph node regions in breast cancer radiation therapy - PROCAB guidelines. Radiother Oncol 2015;114:11-16.

36 Andratschke N, Maurer J, Molls M, et al.: Late radiation-induced heart disease after radiotherapy. Clinical importance, radiobiological mechanisms and strategies of prevention. Radiother Oncol 2011;100:160-166.

37 Galecki J, Hicer-Grzenkowicz J, Grudzien-Kowalska $\mathrm{M}$, et al.: Radiation-induced brachial plexopathy and hypofractionated regimens in adjuvant irradiation of patients with breast cancer - a review. Acta Oncol 2006;45:280-284.
38 Tjessem KH, Johansen S, Malinen E, et al.: Long-term cardiac mortality after hypofractionated radiation therapy in breast cancer. Int J Radiat Oncol Biol Phys 2013;87:337-343.

39 Haviland JS, Owen JR, Dewar JA, et al.: The UK Standardisation of Breast Radiotherapy (START) trials of radiotherapy hypofractionation for treatment of early breast cancer: 10-year follow-up results of two randomised controlled trials. Lancet Oncol 2013;14:10861094

40 Whelan TJ, Pignol J-P, Levine MN, et al.: Long-term results of hypofractionated radiation therapy for breast cancer. N Engl J Med 2010;362:513-520

41 Smith BD, Bentzen SM, Correa CR, et al.: Fractionation for whole breast irradiation: An American Society for Radiation Oncology (ASTRO) evidence-based guideline. Int J Radiat Oncol Biol Phys 2011;81:59-68. 